\title{
Near-Field Interactions between Metal Nanoparticle Surface Plasmons and Molecular Excitons in Thin-Films. Part I: Absorption
}

\author{
Bjoern Niesen, ${ }^{\dagger \ddagger}$ Barry P. Rand, ${ }^{* \dagger}$ Pol Van Dorpe, ${ }^{\dagger, \ddagger}$ David Cheyns, ${ }^{\dagger}$ Honghui Shen, ${ }^{\S}$ Bjorn Maes, ${ }^{\S} \|$ \\ and Paul Heremans ${ }^{\dagger, \ddagger}$ \\ ${ }^{\dagger}$ Imec, Kapeldreef 75, B-3001 Leuven, Belgium \\ ${ }^{\ddagger}$ Department of Electrical Engineering (ESAT), Katholieke Universiteit Leuven, Kasteelpark Arenberg 10, B-3001 Leuven, Belgium \\ ${ }^{\S}$ Photonics Research Group (INTEC), Ghent University-Imec, Sint-Pietersnieuwstraat 41, B-9000 Gent, Belgium \\ "Micro- and Nanophotonic Materials Group, Faculty of Science, University of Mons, Avenue Maistriau 19, B-7000 Mons, Belgium
}

\begin{abstract}
In this and the following paper (parts I and II, respectively), we systematically study the interactions between surface plasmons of metal nanoparticles (NPs) with excitons in thin-films of organic media. In an effort to exclusively probe near-field interactions, we utilize spherical $\mathrm{Ag} \mathrm{NPs}$ in a size-regime where far-field light scattering is negligibly small compared to absorption. In part $\mathrm{I}$, we discuss the effect of the presence of these Ag NPs on the absorption of the embedding medium by means of experiment, numerical simulations, and analytical calculations, all shown to be in good agreement. We observe absorption enhancement in the embedding medium due to the Ag NPs with a strong dependence on the medium permittivity, the spectral position relative to the surface plasmon resonance frequency, and the thickness of the organic layer. By introducing a low-index spacer layer between the NPs and the organic medium, this absorption enhancement is experimentally confirmed to be a near-field effect. In part II, we probe the impact of the Ag NPs on the emission of organic molecules by timeresolved and steady-state photoluminescence measurements.
\end{abstract}

\section{INTRODUCTION}

When excited by light of appropriate wavelength $(\lambda)$, metal nanoparticles (NPs) exhibit so-called localized surface plasmon resonances (LSPRs) due to the collective oscillation of their conduction electrons. ${ }^{1}$ The resonance wavelength $\left(\lambda_{\text {LSPR }}\right)$ and therefore also light absorption and scattering due to LSPRs are highly dependent on the composition, size, shape, and dielectric environment of the NPs. ${ }^{2-8}$ Owing to the strong near-field enhancement originating from the excitation of LSPRs, ${ }^{9,10}$ metal NPs can locally increase the absorption in the embedding medium. In molecular media, the absorption of a photon results in the generation of an exciton due to the transition of an electron from the highest occupied to the lowest unoccupied molecular orbital. The absorption enhancement due to the presence of metal NPs thus increases the amount of excitons generated in the molecular medium. If not dissociated, the excitons will eventually decay either radiatively by emitting photons or nonradiatively by heat dissipation to the environment. The presence of a nearby metal NP can strongly affect the emission quantum yield by introducing additional nonradiative decay channels and enhancing the radiative decay rate. ${ }^{11-13}$ These plasmon-exciton interactions, together with the sensitivity of the LSPR to the dielectric environment, give metal nanoparticle-organic molecular systems potential applications in organic and dye-sensitized photovoltaic cells, ${ }^{14-19}$ organic light emitting devices, ${ }^{20,21}$ chemical and biological sensors, ${ }^{22-24}$ surface enhanced spectroscopy, ${ }^{25,26}$ photochemistry, ${ }^{27}$ and lasers. ${ }^{28,29}$

Recently, there has been a significant effort dedicated to surface plasmon-exciton interactions. Especially, their effect on the absorption ${ }^{30-33}$ and photoluminescence ${ }^{33-36}$ of organic molecules has been extensively studied. Despite the substantial research activity on this topic, a systematic study is still missing that combines experiments, simulations, and analytical calculations to individually examine the effect of near-field LSPR-exciton interactions on the absorption and emission of molecular thin-films. In particular, the influence of the dispersive permittivity of strongly absorbing molecular thinfilms has not been studied thoroughly. In addition, only a few studies exclusively characterize the near-field plasmon-exciton interactions using metal NPs that exhibit negligible far-field scattering.

In this and the subsequent paper (parts I and II, respectively), we utilize spherical metal NPs in a size-regime where far-field scattering is negligibly small compared to absorption, to systematically probe near-field plasmon-exciton 

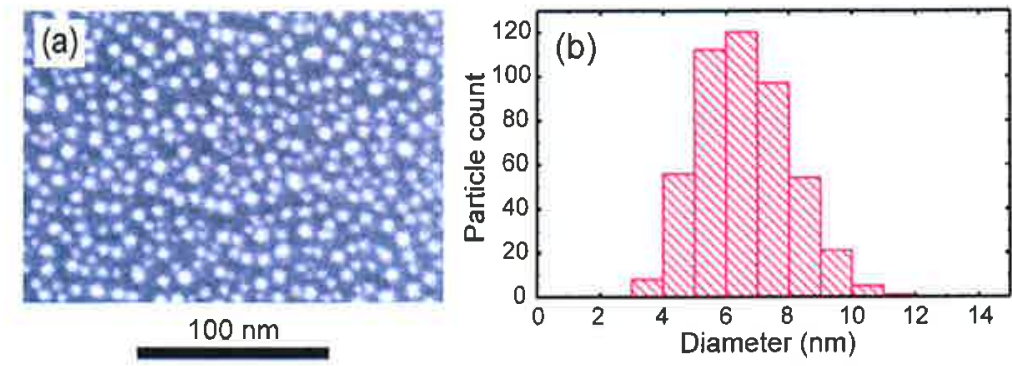

Figure 1. (a) Top-view scanning electron micrograph of the $\mathrm{Ag} \mathrm{NP}$ layer on a $\mathrm{Si} / \mathrm{SiO}_{2}$ substrate. (b) Histogram of the Ag NP size-distribution obtained from 474 particles found in the micrograph. The average diameter is $6.6 \mathrm{~nm}$, with a standard deviation of $1.4 \mathrm{~nm}$.

interactions by means of absorption as well as steady-state and time-resolved photoluminescence spectroscopy. We employ a model system of a Ag NP layer on a transparent substrate that is covered by a thin-film of a molecular medium either in direct contact with the Ag NPs or separated from them by a transparent, nonemissive spacer layer. In part I, we examine the effect of the Ag NP layer on the absorption of organic molecules. We observe absorption enhancement in the presence of the NPs with a strong dependence on the thickness of the organic layer and the wavelength of incident light. Numerical simulations show good quantitative agreement with experiment, which we could also reproduce qualitatively by an analytical model. The near-field nature of the absorption enhancement is experimentally confirmed by introducing a spacer layer between the Ag NP layer and the organic medium. Finally, we observe a strong dependence of the absorption enhancement on the dielectric environment of the Ag NPs. In part $\mathrm{II},{ }^{37}$ we analyze the impact of the Ag NP layer on the emission of organic molecules by steady-state and time-resolved photoluminescence measurements. These experimental results are then discussed utilizing exciton decay rates obtained by an analytical model.

\section{EXPERIMENTAL METHODS}

Copper(II) phthalocyanine (CuPc) was purchased from Sigma-Aldrich, and chloroboron subphtalocyanine (SubPc) was purchased from the Luminescence Technology Corp. Both materials were purified twice by thermal gradient sublimation prior to use. Hyflon AD60X, a copolymer of 2,2,4-trifluoro-5trifluorometoxy-1,3-dioxole and tetra-fluoro-ethylene with a molar ratio of 0.6:0.4, was obtained from Solvay-Solexis and used as received. Silver with a purity of $99.99 \%$ was purchased from the Kurt. J. Lesker Company. Eagle XG glass (purchased from Corning Inc.) and $\mathrm{Si} / \mathrm{SiO}_{2}$ substrates were cleaned by subsequent sonication in soapy water, deionized water, acetone, and isoproyl alcohol, followed by exposure to UV-ozone for 15 min. Silver, $\mathrm{CuPc}$, and $\mathrm{SubPc}$ were deposited by thermal evaporation at a chamber pressure below $2 \times 10^{-6}$ Torr. Silver was deposited at a rate of $0.01 \mathrm{~nm} / \mathrm{s}$; $\mathrm{CuPc}$ and SubPc were deposited at a rate of $0.1 \mathrm{~nm} / \mathrm{s}$. Hyflon AD60X thin-films were deposited in the same chamber by means of vacuum pyrolysis, ${ }^{38}$ at a pressure below $5 \times 10^{-6}$ Torr and a rate of $0.05 \mathrm{~nm} / \mathrm{s}$. During all depositions, the rate and film thickness were monitored by a quartz-crystal oscillator. The substrate was kept at room temperature and was rotating during the deposition, leading to a thickness inhomogeneity of $<5 \%$ over an area of $100 \mathrm{~cm}^{2}$, as determined by spectroscopic ellipsometry. A computer-controlled retractable shadow mask allowed for the deposition of layers with six different thicknesses in a single run.

The specular light transmission of thin-films on glass substrates was measured using a Shimadzu UV-1601PC spectrophotometer, whereas the total (i.e., the sum of specular and diffuse) transmission and reflectance were measured using a Bentham PVE 300 photovoltaic device characterization system equipped with an integrating sphere. As far-field light scattering by the thin-films with and without Ag NPs was found to be negligible (see the Supporting Information, Figure S1), the light absorption was defined as absorption $=1-$ specular transmission - total reflectance. The reason for using the specular instead of the total transmission was the significantly higher noise level in the total transmission spectra due to the integrating sphere. The absorption of the glass substrate was subtracted from all absorption spectra. The inaccuracy of the absorption spectra of samples deposited during a single run, resulting from the thickness inhomogeneity and the inaccuracy of the measurement set-ups, was estimated as $\pm 0.5 \%$ for $\lambda>$ $350 \mathrm{~nm}$ and $\pm 2 \%$ for $\lambda<350 \mathrm{~nm}$ (see the Supporting Information, Figure S2). The morphology of the Ag NP layer was determined by a Hitachi SU8000 scanning electron microscope. The size-distribution of the $\mathrm{Ag}$ NPs was determined from scanning electron micrographs using Gwyddion 2.18. The relative permittivity, $\varepsilon=\varepsilon_{1}+i \varepsilon_{2}$, of all materials was determined from their thin-films on $\mathrm{Si} / \mathrm{SiO}_{2}$ substrates, using a SOPRA GESP-5 spectroscopic ellipsometer.

\section{RESULTS AND DISCUSSION}

3.1. Ag NP Layer. The deposition of a Ag layer with a nominal thickness of $1 \mathrm{~nm}$ on a glass substrate by means of thermal evaporation spontaneously leads to the formation of a dense Ag NP layer. As shown by the top-view scanning electron micrograph in Figure 1a, the $\mathrm{Ag}$ NPs approximately have a circular shape. The particle diameter distribution obtained from this micrograph has average and maximum values of 6.6 and 12 $\mathrm{nm}$, respectively, with a standard deviation of $1.4 \mathrm{~nm}$ (Figure 1b). From atomic force microscopy measurements, ${ }^{39}$ an average height of $5 \mathrm{~nm}$ was obtained. The absorption spectrum of the bare Ag NP layer, shown as a black dotted curve in Figure 2a, exhibits a single absorption band centered at $\lambda=425$ $\mathrm{nm}$, which originates from a dipole LSPR. ${ }^{39}$ As expected for NPs of this size, there are no additional absorption bands due to higher order modes. ${ }^{40}$ The absorption band extending from $\lambda=300-320 \mathrm{~nm}$ originates from $\mathrm{Ag}$ interband transitions. ${ }^{41}$

In general, when describing the light extinction of metal NPs, both absorption as well as light scattering have to be taken into account. The extinction is then given by the sum of absorption and scattering. According to electrodynamic Mie theory, ${ }^{42}$ for 


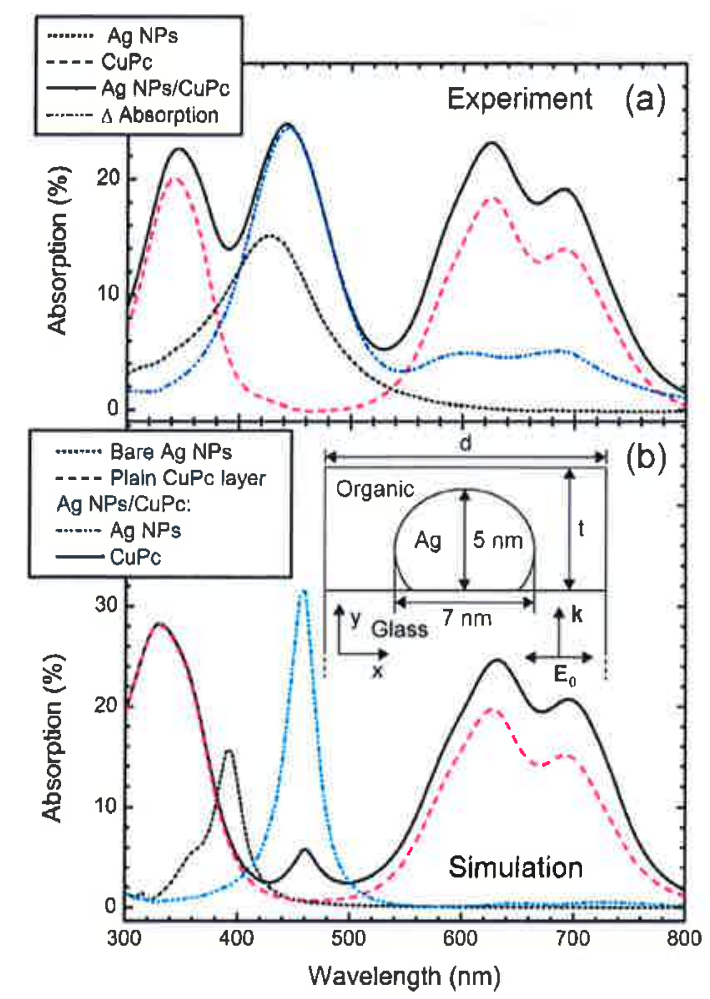

Figure 2. (a) Absorption spectra of the bare Ag NP layer (dotted black curve), the Ag NP layer coated by $10 \mathrm{~nm}$ of CuPc (solid black curve), and a $10 \mathrm{~nm}$ thick CuPc layer on glass (dashed red curve). The absorption difference between the CuPc layers with and without $\mathrm{Ag}$ NPs is shown as a blue dash-dot-dot curve. (b) Results obtained from three-dimensional numerical simulations using a geometry with a cross section as shown in the inset. Periodic boundaries in both lateral dimensions result in an infinite two-dimensional array of truncated $\mathrm{Ag}$ spheroids with center-to-center spacing $d$. The light wave is incident through the glass substrate with the propagation vector, $\mathbf{k}$, and electric field vector, $\mathbf{E}_{0}$, as indicated by arrows. Further details of the simulation are given in the text. This graph shows the simulated absorption in the bare Ag NPs (dotted black curve), a pure $10 \mathrm{~nm}$ thick $\mathrm{CuPc}$ layer (dashed red curve), a CuPc layer covering the $\mathrm{Ag}$ NPs with $t$ equivalent to the pure CuPc layer (solid black curve), and the Ag NPs when covered by that layer (green dash-dot-dot curve). For this simulation, $d$ was set to $18 \mathrm{~nm}$.

spherical Ag NPs with a diameter of less than $12 \mathrm{~nm}$ in air, the scattering-to-extinction ratio remains below $7 \times 10^{-3}$ (see the Supporting Information, Figure S3). This ratio increases with increasing relative permittivity of the (nonabsorbing) embedding medium $\left(\varepsilon_{\mathrm{m}}\right)$ and reaches values of up to 0.16 for $\mathcal{E}_{\mathrm{m}}=10$. Light scattering thus only becomes significant for the extreme cases of the largest particles in the NP layer and very high $\varepsilon_{\mathrm{m}}$. Recently, a strong influence of light scattering in absorbing media even for small spherical Ag NPs was predicted from an extended Mie theory. ${ }^{43}$ However, far-field light scattering was found to be negligible for the Ag NP layer employed here, even when it was coated with absorbing organic media (see the Supporting Information, Figure S1). Throughout this part of the study, the light extinction will therefore be approximated by the absorption, and far-field scattering will be neglected. As we will see later, this approximation does not hold when discussing the effect of the Ag NPs on light emission in part II. ${ }^{37}$
3.2. Absorption Enhancement in CuPc. We chose $\mathrm{CuPc}$, a well-characterized organic semiconductor, as a representative material to study the effect of the Ag NP layer on the absorption of organic molecules. A major advantage of this material choice is the complementary nature of the absorption spectra of $\mathrm{CuPc}$ and the Ag NP layer. The absorption spectrum of a $10 \mathrm{~nm}$ thick $\mathrm{CuPc}$ layer on glass is shown as a red dashed curve in Figure 2a. It features two absorption bands: the Soret band centered at $\lambda=345 \mathrm{~nm}$ and the low energy $Q$ band with peaks at $\lambda=625$ and $690 \mathrm{~nm}$. The absorption spectrum of 10 $\mathrm{nm} \mathrm{CuPc}$ deposited on the Ag NP layer (black solid curve) exhibits the same features as well as the LSPR absorption band of the Ag NPs. The LSPR is slightly red-shifted to $\lambda=450 \mathrm{~nm}$ compared to that of the bare $\mathrm{Ag}$ NP layer due to the change in the dielectric environment. ${ }^{4,45}$ The absorption difference between the sample with and without Ag NPs is shown as a blue curve. It features a band at $550 \mathrm{~nm}<\lambda<800 \mathrm{~nm}$ that is not present in the absorption spectrum of the uncovered $\mathrm{Ag}$ NP layer. This increase in absorption has been attributed to an absorption enhancement in the $\mathrm{CuPc}$ medium caused by the $\mathrm{Ag}$ NP LSPR ${ }^{30,43}$ In the following paragraphs, this absorption enhancement will be characterized in detail, and it will be discussed whether it exclusively originates from the Ag NP LSPR

To this end, three-dimensional (3D) finite-element numerical simulations were made. The geometry used for these simulations was motivated by our microscopy study (see Figure 1). Its cross section is shown as an inset to Figure $2 \mathrm{~b}$. The $\mathrm{Ag}$ NPs are represented by truncated spheroids with a height of 5 $\mathrm{nm}$ and a lateral dimension of $7 \mathrm{~nm}$. Periodic boundary conditions in the lateral directions lead to a $\mathrm{Ag} \mathrm{NP}$ array with a center-to-center spacing denoted by $d$. An organic layer with a thickness $t$ and a flat surface covers the Ag NPs. The light is simulated by a plane wave with the propagation vector, $\mathrm{k}$, parallel to the $y$ axis and the electric field vector, $\mathbf{E}_{0}$, parallel to the $x$ axis. It is incident through the semi-infinite glass substrate with $\varepsilon=2.25$. The $\varepsilon$ values used for these simulations are shown in the Supporting Information, Figure S4. As a reference, neat organic films without Ag NPs were simulated. When the simulation results from such a reference film are compared to those of an organic film on Ag NPs, the thickness of the film on Ag NPs is chosen slightly larger than that of the neat reference film such that both organic layers have the same volume, compensating for the volume occupied by the Ag NPs.

The software package used for these simulations, COMSOL Multiphysics 3.5a, allowed us to monitor the absorption in each component individually and therefore to deconstruct the absorption spectra of composite layers as shown in Figure $2 \mathrm{~b}$. The simulated absorption of a pure $10 \mathrm{~nm}$ thick $\mathrm{CuPc}$ layer and that of a CuPc layer with equivalent $t$ covering the Ag NPs are shown as dashed red and solid black curves, respectively. The absorption in the $\mathrm{Q}$ band is significantly enhanced in the presence of the Ag NPs, whereas the Soret band remains unchanged. The simulated absorption in the Ag NPs when covered by $\mathrm{CuPc}$ (green dash-dot-dot curve) exhibits a sharp peak at $\lambda=460 \mathrm{~nm}$, red-shifted compared to the absorption peak of the uncovered NPs at $\lambda=390 \mathrm{~nm}$ (dotted black curve). In addition, it also features a very weak absorption band in the $\mathrm{CuPc} \mathrm{Q}$ band spectral region, which is absent for the uncovered NPs.

Combined, these simulated spectra of the individual components accurately describe the experimentally obtained spectra shown in Figure 2a, including the absence of absorption 
enhancement at $\lambda<\lambda_{\mathrm{LSPR}}$. Moreover, these results suggest that most of the absorption in the spectral region of the $\mathrm{CuPc} Q$ band occurs within the organic medium and not in the NPs, which is in line with previously reported results from analytical and numerical calculations. ${ }^{43}$ The slight mismatch of $\lambda_{\text {LSPR }}$ and the narrower absorption band compared to the experimentally observed results can be explained by the uniform size and shape of the simulated particles and the uncertainty about the exact NP shape.

In order to determine the range of the absorption enhancement, samples with various $\mathrm{CuPc}$ layer thicknesses were fabricated. The absorption spectra of the samples with and without Ag NPs, shown in Figure 3a as solid and dashed curves,
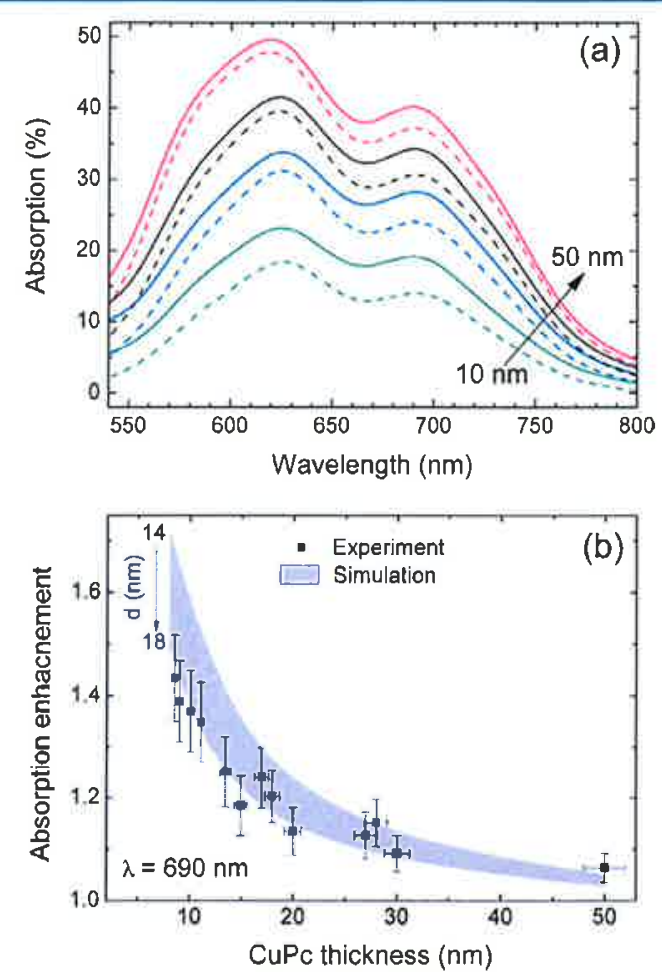

Figure 3. (a) Absorption spectra of CuPc layers deposited on glass (dashed curves) or on the Ag NP layer (solid curves) for $\mathrm{CuPc}$ layer thicknesses of 10,20,30, and $50 \mathrm{~nm}$. (b) Absorption enhancement due to the Ag NP layer at $\lambda=690 \mathrm{~nm}$ determined by dividing the absorption of the Ag NP layer coated with CuPc by that of pure $\mathrm{CuPc}$ layers for $\mathrm{CuPc}$ layer thicknesses between 8 and $50 \mathrm{~nm}$ (black squares). The gray area shows the absorption enhancement at the same wavelength and for the same CuPc thickness range obtained by dividing the simulated absorption in the $\mathrm{CuPc}$ layer covering the $\mathrm{Ag}$ $\mathrm{NPs}$ by that of a $\mathrm{CuPc}$ layer on glass with equivalent $t$. Center-tocenter spacing $(d)$ values of 14 and $18 \mathrm{~nm}$ were used for these simulations.

respectively, clearly show a decreasing influence of the Ag NPs with increasing $\mathrm{CuPc}$ layer thickness. The absorption enhancement at $\lambda=690 \mathrm{~nm}$ obtained from these measurements is shown in Figure $3 \mathrm{~b}$ as black squares. It decreases from $1.44 \pm$ 0.08 for a $8 \mathrm{~nm}$ thick CuPc layer to $1.06 \pm 0.03$ for a $50 \mathrm{~nm}$ thick layer. This experimentally measured thickness dependence could be reproduced by numerical simulations (gray area in Figure 3b). Quantitative agreement was obtained when $d$ was set to $18 \mathrm{~nm}$ for the thinnest and to $14 \mathrm{~nm}$ for the thickest
$\mathrm{CuPc}$ layer. Both these values are in line with typical center-tocenter distances observed in Figure 1a. A possible cause for the deviation between experiment and simulation could be the roughness of the $\mathrm{CuPc}$ layer surface, which is not taken into account in the simulations and becomes increasingly important for thinner layers.

The absorption enhancement obtained by these simulations is equivalent to the enhancement of the $\mathbf{E}$ field intensity due to the Ag NPs, $\eta=\left|E_{N P}\right|^{2} /\left|E_{0}\right|^{2}$, where $E_{0}$ and $E_{N P}$ denote the $E$ field without NPs and in their presence, respectively. The contour-plot of $\eta$ at $\lambda=690 \mathrm{~nm}$ in the $x-y$ plane cutting through the center of the simulated Ag NP therefore yields direct information about the range of the absorption enhancement (see Figure $4 a$ ). The absorption is strongly enhanced
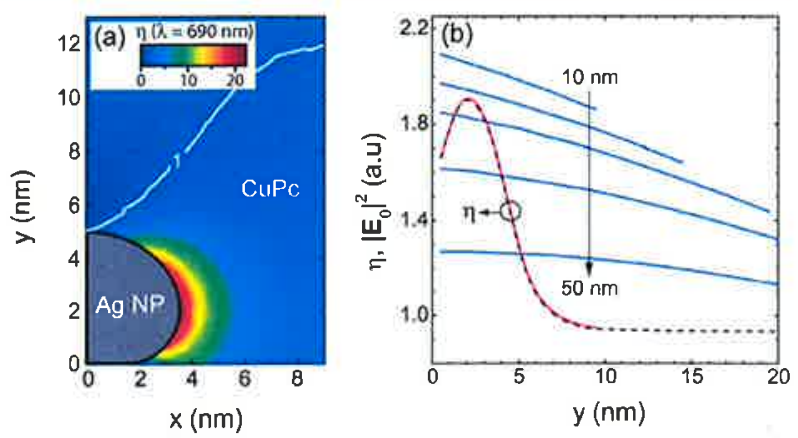

Figure 4. (a) Simulated $\eta$ around a Ag NP when covered by a $50 \mathrm{~nm}$ thick $\mathrm{CuPc}$ layer. (b) Simulated average $\eta$ value integrated over a slice of $\mathrm{CuPc}$ medium in the $x-z$ plane versus the height along the $y$ axis for a $10 \mathrm{~nm}$ (red solid curve) and $50 \mathrm{~nm}$ (dashed black curve) thick layer. The solid blue curves show the simulated $\left|\mathbf{E}_{0}\right|^{2}$ versus the $y$ axis in neat CuPc layers with thicknesses of $10,15,20,30$, and $50 \mathrm{~nm}$. $d$ was set to $18 \mathrm{~nm}$ for all simulations shown in this figure. The simulation results shown here were obtained at $\lambda=690 \mathrm{~nm}$.

between the NPs, whereas it is reduced above the NPs as evidenced by $\eta<1$. The border between enhanced and reduced absorption (the $\eta=1$ contour) extends from the top of the NP to the border of the simulation halfway to the neighboring NP where it levels off at a height of $12 \mathrm{~nm}$. The very large field enhancement close to the Ag NP surface observed in this graph might possibly not contribute to absorption enhancement in real-world experiments as the distance between a $\mathrm{Ag}$ surface and the first $\mathrm{CuPc}$ monolayer is about $0.3 \mathrm{~nm} .{ }^{46}$ However, as the exact morphology of the $\mathrm{Ag} \mathrm{NP}-\mathrm{CuPc}$ interface is not known and the volume of the void is very small compared to the total $\mathrm{CuPc}$ layer volume, we did not take this metalmolecule separation into account, which means that the simulated absorption enhancement will be slightly overestimated. Figure $4 \mathrm{~b}$ shows the average $\eta$ value integrated over a slice of CuPc medium in the $x-z$ plane versus the height along the $y$ axis for CuPc layer thicknesses of $10 \mathrm{~nm}$ (solid red curve) and $50 \mathrm{~nm}$ (dashed black curve). $\eta$ is nearly identical for both layer thicknesses, with $\eta>1$ up to a height of $7 \mathrm{~nm}$. Above this height, $\eta$ converges to a value of 0.94 .

These simulation results allow us to explain the experimentally observed $\mathrm{CuPc}$ layer thickness dependence shown in Figure 3. As only the first $7 \mathrm{~nm}$ of the CuPc medium benefit from the absorption enhancement, increasing the $\mathrm{CuPc}$ layer thickness beyond that height will lead to a reduction in absorption enhancement relative to the total absorption of the layer. Moreover, $\left|\mathrm{E}_{0}\right|^{2}$ decreases with increasing $\mathrm{CuPc}$ layer 
thickness (blue curves in Figure 4b), which means that, for the same $\eta$ value, $\left|\mathbf{E}_{\mathrm{Np}}\right|^{2}-\left|\mathbf{E}_{0}\right|^{2}$ and thus also the absorption difference due to the Ag NPs will be smaller for thicker layers.

A rather subtle feature of the absorption enhancement can be observed when analyzing the $\mathrm{CuPc} Q$ band spectral region (550 $\mathrm{nm}<\lambda<800 \mathrm{~nm}$ ) of the absorption spectra shown in Figure 2 . The normalized experimentally measured absorption spectra of the samples with and without Ag NPs are shown in Figure 5 as

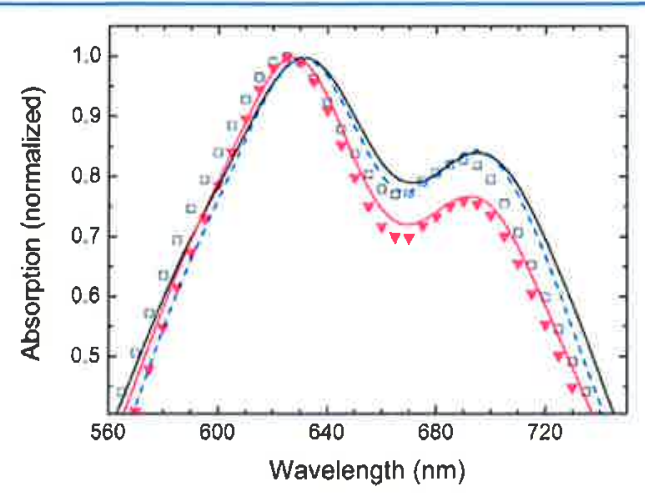

Figure 5. Normalized measured absorption spectra of $10 \mathrm{~nm} \mathrm{CuPc}$ deposited on glass (solid red triangles) and on the Ag NP layer (open black squares), normalized simulated absorption in a $10 \mathrm{~nm}$ thick $\mathrm{CuPc}$ layer on glass (solid red curve) and in a $\mathrm{CuPc}$ layer with equivalent $t$ covering the Ag NPs (solid black curve), and the absorption spectrum obtained by normalizing the product of the experimentally measured absorption of $10 \mathrm{~nm} \mathrm{CuPc}$ on glass and $\eta_{\max }$ for a $\mathrm{Ag} \mathrm{NP}$ in $\mathrm{CuPc}$ obtained by eq 2 (dashed blue curve). The values of $r$ and $R$ used to calculate $\eta_{\max }$ are not important as the results were finally normalized. black squares and red triangles, respectively. The normalized simulated spectra are shown as solid lines. The relative intensities of the peaks at $\lambda=690 \mathrm{~nm}$ in the experimental and simulated spectra are enhanced by a similar amount in the presence of the Ag NPs. Considering the experimental results only, this change in relative peak intensity might be interpreted as a change in the $\mathrm{CuPc}$ layer morphology when grown on the Ag NP substrate, as this ratio is an indicator of the degree of molecular aggregation within the $\mathrm{CuPc}$ layer. ${ }^{47} \mathrm{An}$ increased relative intensity of the peak at $\lambda=690 \mathrm{~nm}$ might indicate a decrease in $\mathrm{CuPc}$ aggregation. However, the numerical simulations, that do not take into account any change in the interactions between the $\mathrm{CuPc}$ molecules or between the molecules and the substrate when introducing the NPs, accurately reproduce this change in relative peak intensities. Therefore, we conclude that the spectral shape of the absorption enhancement can be reproduced by only taking into account the wavelength-dependent enhancement of the electromagnetic field intensity around the NPs.

In addition to numerical simulations, the absorption enhancement due to the Ag NPs can also be described analytically. Because of the small Ag NP size relative to the wavelengths of the measured spectra, the $\mathrm{E}$ field across an illuminated NP is approximately constant and the so-called quasi-static approximation can be employed. According to this approximation, the dipole polarizability, $\alpha(\omega)$, of a spherical metal NP with radius $R$ in a homogeneous medium is given by ${ }^{1}$

$$
\alpha(\omega)=4 \pi R^{3} \frac{\varepsilon_{\mathrm{NP}}(\omega)-\varepsilon_{\mathrm{m}}(\omega)}{\varepsilon_{\mathrm{NP}}(\omega)+2 \varepsilon_{\mathrm{m}}(\omega)}
$$

where $\omega$ denotes the frequency of the incident electromagnetic wave and $\varepsilon_{\mathrm{NP}}$ is the relative permittivity of the metal NP. In a

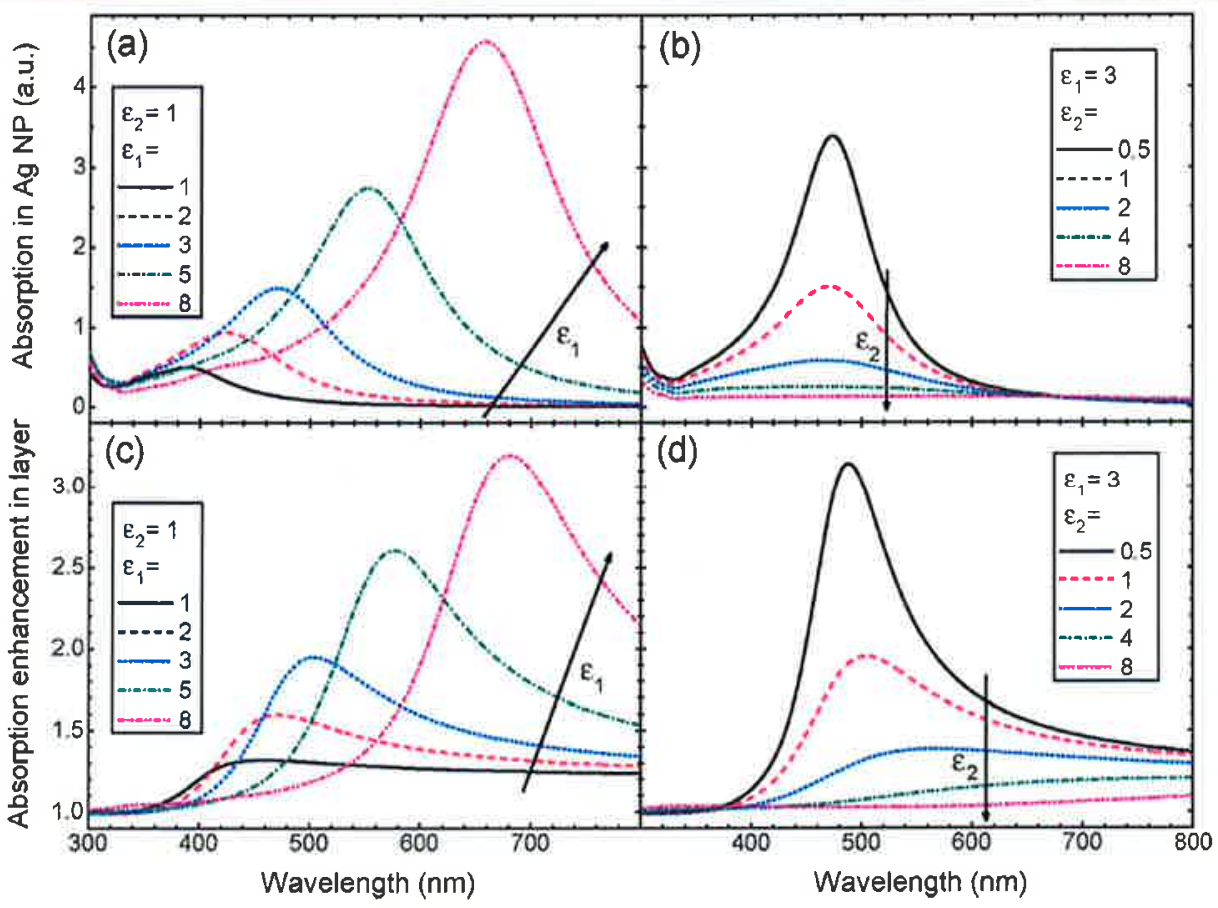

Figure 6. (a, b) Simulated absorption in the Ag NPs when covered by a layer with a volume identical to that of a $10 \mathrm{~nm}$ thick neat layer for various (a) $\varepsilon_{1}$ and (b) $\varepsilon_{2}$. (c, d) Simulated absorption enhancement in the layer covering the Ag NPs compared to a $10 \mathrm{~nm}$ thick plain layer. $d$ was set to 18 $\mathrm{nm}$ for all these simulations. 
nonabsorbing medium, the maximal $\mathbf{E}$ field intensity enhancement, $\eta_{\max }(\omega, r)$, at the distance $r$ to the center of the NP is given by ${ }^{1,48}$

$$
\eta_{\max }(\omega, r)=\left|1+\frac{\alpha(\omega)}{2 \pi r^{3}}\right|^{2}
$$

As eq 2 is only exact for nonabsorbing media, $\eta_{\max }$ can merely be considered as an upper limit for the $\mathbf{E}$ field enhancement in absorbing media, where an accurate treatment requires employing an electrodynamic formalism. ${ }^{43,49}$ For this reason, and because it assumes a spherical geometry, eq 2 will only yield qualitative information when applied to the thin-film system studied here. We calculated $\eta_{\max }$ for Ag NPs embedded in $\mathrm{CuPc}$ by inserting the $\varepsilon$ values of $\mathrm{Ag}$ and $\mathrm{CuPc}$ shown in Figure S4 of the Supporting Information into eqs 1 and 2 . The resulting value was then multiplied with the experimentally measured absorption spectrum of the neat $10 \mathrm{~nm}$ thick $\mathrm{CuPc}$ layer and finally normalized. This calculated absorption spectrum is shown as a dashed blue curve in Figure 5. It is nearly identical to the simulated spectrum of the composite layer and thus also well in agreement with the experimental data. This means that the spectral shape of the absorption enhancement in the $\mathrm{CuPc} \mathrm{Q}$ band is directly related to $\varepsilon_{\mathrm{NP}}$ and $\varepsilon_{\mathrm{m}}$. This also suggests that the influence of small deviations of the NP shape from a perfect sphere as well as particlesubstrate and particle-particle interactions are negligible. The limited influence of the substrate can be explained by its low $\varepsilon$ compared to that of $\mathrm{CuPc}$ in this spectral region, such that the LPSR is more strongly affected by the organic medium. The negligible contribution of particle-particle interactions is expected for a $\mathrm{Ag} \mathrm{NP}$ layer where the distance between the NPs is similar to their diameter, as such interactions would only become relevant for denser NP layers. ${ }^{50}$

3.3. Spectral Dependence and Influence of $\varepsilon$. After having confirmed the validity of numerical simulations to describe the absorption enhancement in $\mathrm{CuPc}$, we can extend these simulations to other embedding materials with spectrally independent $\varepsilon=\varepsilon_{1}+i \varepsilon_{2}$, employing the same geometry as used for CuPc. Figure 6a and b shows the simulated absorption in the Ag NPs when covered by these various materials. When $\varepsilon_{2}$ of the material is set to 1 and $\varepsilon_{1}$ is altered from 1 to 8 , the LSPR absorption peak of the Ag NPs red-shifts from $\lambda=390$ to 660 $\mathrm{nm}$ while its intensity increases by almost 1 order of magnitude (see Figure 6a). For constant $\varepsilon_{1}=3$, a typical value for many organic materials, an increase in $\varepsilon_{2}$ dampens the resonance intensity such that the absorption peak completely vanishes for $\varepsilon_{2} \geq 4$, whereas the resonance frequency is not affected (see Figure $6 \mathrm{~b}$ ). The simulated absorption enhancement in these embedding media due to the Ag NPs is shown in Figure $6 \mathrm{c}$ and d. It scales with the intensity of the LSPR and is strongest at $\lambda$ slightly larger than $\lambda_{\text {LSPR }}$. For this set of materials, values of up to 3.2 are reached for $\varepsilon=3+0.5 i$ and $\varepsilon=8+1 i$. For all of these materials, the enhancement steeply decreases at $\lambda<\lambda_{\mathrm{LSPR}}$, whereas at $\lambda>\lambda_{\text {LSPR }}$ it exhibits an extended tail with values above unity up to $\lambda=800 \mathrm{~nm}$. For highly absorbing media $\left(\varepsilon_{2}\right.$ $\geq 2$ ), the absorption enhancement only reaches values below 1.5 , with an off-resonance enhancement that is comparable or even stronger than that at $\lambda_{\text {LSPR. }}$. This strong dependence of the absorption enhancement on $\varepsilon_{2}$ is consistent with previously reported results obtained using an analytical model. ${ }^{51}$ In summary, the strongest absorption enhancement is thus reached for materials with a large $\varepsilon_{1}$ and a weak absorption, resulting in a small $\varepsilon_{2}$. Typically, the enhancement is strongest slightly red-shifted to $\lambda_{\text {LSPR. }}$. However, exploiting the offresonance enhancement rather than the stronger enhancement at $\lambda_{\mathrm{LPR}}$ can still be beneficial as the absorption in the Ag NPs is much smaller far away from the resonance. This effect is especially relevant for applications where reducing parasitic absorption of the metal NPs is advantageous. The spatial distribution of the absorption enhancement was found to only weakly depend on the spectral position, as shown for $\varepsilon=3+$ $0.5 i$ in the Supporting Information, Figure S5. Off-resonance as well as at $\lambda_{\text {LPR }}$ the absorption enhancement is limited to a height of $\sim 7 \mathrm{~nm}$, such that the stronger enhancement at $\lambda_{\mathrm{LSPR}}$ is mainly due to higher $\mathbf{E}$ field intensities in the interstices of the NPs.

We can apply the knowledge gained from these simulation results to explain the spectral shape of the $\mathrm{CuPc} Q$ band absorption enhancement. For a material with constant $\varepsilon \approx 3+$ $2 i$, the absorption enhancement is expected to slightly decrease with increasing $\lambda$ for $\lambda>\lambda_{\mathrm{LSPR}}$. However, in the case of $\mathrm{CuPc}$, this decrease is outweighed by a larger $\varepsilon_{1}$ and smaller $\varepsilon_{2}$ at $\lambda=$ $690 \mathrm{~nm}$ compared to the $\varepsilon$ at $\lambda=625 \mathrm{~nm}$, leading to a stronger absorption enhancement at $\lambda=690 \mathrm{~nm}$ (see the Supporting Information, Figure S4). This effect is even more pronounced for $\mathrm{SubPc}$, a material with a highly dispersive $\varepsilon$. When deposited on the Ag NP layer, the relatively low value of $\varepsilon_{1}$ at $400 \mathrm{~nm}<\lambda<550 \mathrm{~nm}$ results in the excitation of a LSPR at $\lambda=$ $445 \mathrm{~nm}$. Due to the narrow and intense absorption band of SubPc at $\lambda=590 \mathrm{~nm}$ (dashed black curve in Figure 7), $\varepsilon_{1}$

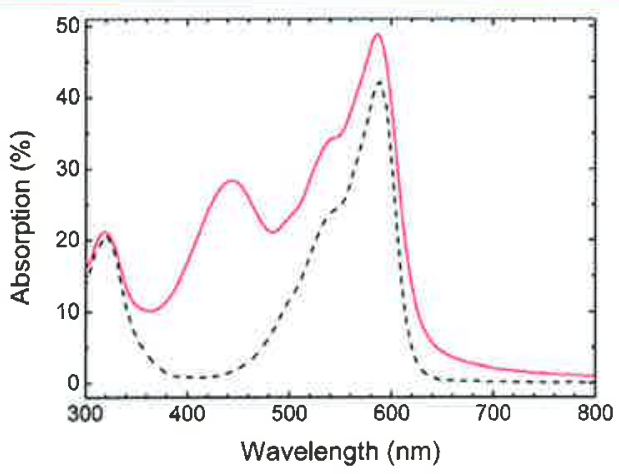

Figure 7. Absorption spectra of $10 \mathrm{~nm}$ thick layers of SubPc deposited on glass (dashed black curve) and on the Ag NP layer (solid red curve).

steeply increases at $\lambda=570-600 \mathrm{~nm}$ and reaches values of up to 10.5. This leads to the excitation of a second dipole LSPR at $\lambda=610 \mathrm{~nm}$, as reported previously. ${ }^{39}$ These two LSPR are evidenced by an additional absorption band peaking at $\lambda=445$ $\mathrm{nm}$, as well as an extension of the red tail of the SubPc absorption band centered at $\lambda=590 \mathrm{~nm}$ (solid red curve).

3.4. Effect of a Spacer Layer. We studied the effect of a spacer layer on the plasmon-exciton interactions for both $\mathrm{CuPc}$ and SubPc by introducing a layer of Hyflon AD60X between the Ag NP layer and the organic medium. Thin-films of Hyflon AD60X were found to be completely transparent in the measured spectral range. Moreover, because of the small $\varepsilon_{1}$ of Hyflon AD60X, the LSPR of the Ag NPs was only slightly altered when covered with it (see the Supporting Information, Figures S4 and S6). We fabricated samples without a spacer and ones that had spacer layers with thicknesses of 1-20 nm. For each spacer layer thickness, we fabricated a sample with and 
one without $\mathrm{Ag}$ NPs. For the reference samples without $\mathrm{Ag}$ NPs, Hyflon AD60X was directly deposited on the glass substrate. The spectra of the absorption differences between the samples with and without Ag NPs are shown in Figure 8a and b for $10 \mathrm{~nm}$ thick layers of $\mathrm{CuPc}$ and SubPc, respectively.

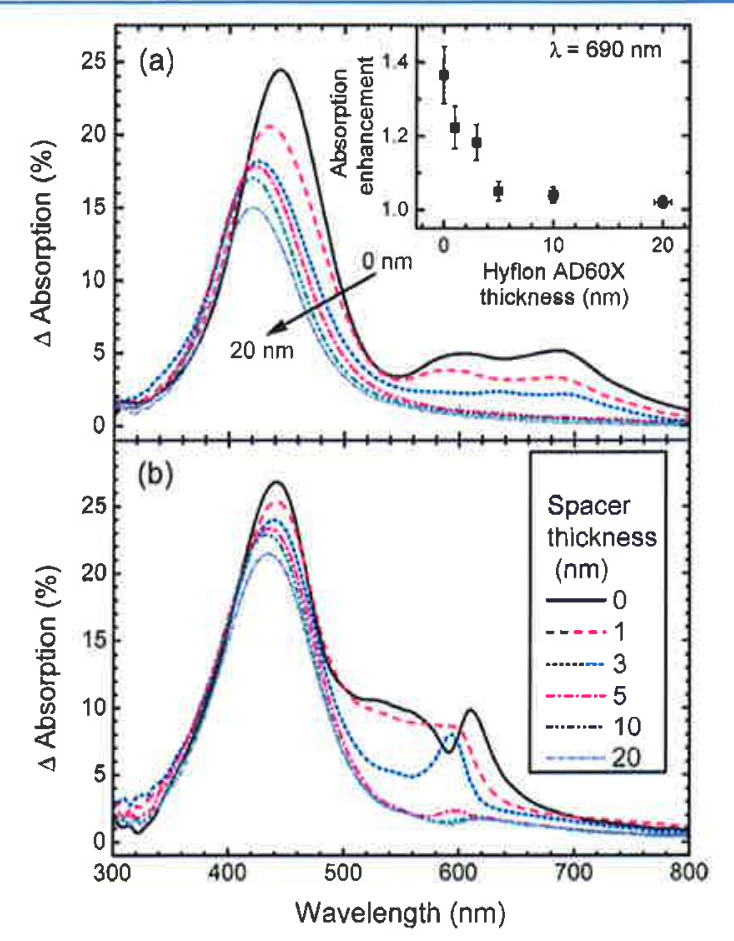

Figure 8. Absorption differences between samples with and without Ag NPs. These samples were made by depositing Hyflon AD60X layers with thicknesses of $1,3,5,10$, and $20 \mathrm{~nm}$ either on the Ag NP layer or on glass, followed by a $10 \mathrm{~nm}$ thick layer of either (a) CuPc or (b) SubPc. For both $\mathrm{CuPc}$ and $\mathrm{SubPc}_{\mathrm{b}}$, the absorption differences of samples without a Hyflon AD60X layer are shown as black curves. The inset to (a) shows the absorption enhancement ratio at $\lambda=690 \mathrm{~nm}$ versus the Hyflon AD60X layer thickness for the CuPc samples.

The absorption difference in the $\mathrm{CuPc} Q$ band rapidly decreases with increasing spacer layer thickness and finally vanishes for a $5 \mathrm{~nm}$ thick spacer, as shown by Figure 8a. For spacer layer thicknesses of $5-20 \mathrm{~nm}$, the absorption differences thus resemble the absorption curve of the Ag NP layer covered only by Hyflon AD60X. The absorption enhancement at $\lambda=$ $690 \mathrm{~nm}$ shown in the inset quantifies this rapid decrease, which is much steeper than that observed for an increasing $\mathrm{CuPc}$ layer thickness without spacer layer (see Figure 3b). The SubPc samples (see Figure $8 \mathrm{~b}$ ) also show a vanishing absorption difference for spacer layers thicknesses beyond $3 \mathrm{~nm}$ but with a notably different behavior for the three thinnest spacer layers compared to the $\mathrm{CuPc}$ samples. In particular, the peak observed at $\lambda=610 \mathrm{~nm}$ without a spacer layer gives way to a shoulder at $\lambda=600 \mathrm{~nm}$ and a peak at $\lambda=595 \mathrm{~nm}$ for Hyflon AD60X layer thicknesses of 1 and $3 \mathrm{~nm}$, respectively.

In order to gain a better understanding of these results, we conducted numerical simulations using the same geometry as shown in the inset to Figure $2 \mathrm{~b}$ but with an additional conformal Hyflon AD60X layer separating the Ag NPs from the SubPc layer. The simulated absorption in the Ag NPs and the increase in absorption in the SubPc layer caused by the NPs are shown in Figure 9a and $b$, respectively. Without the spacer layer, the absorption in the Ag NPs shows two bands centered

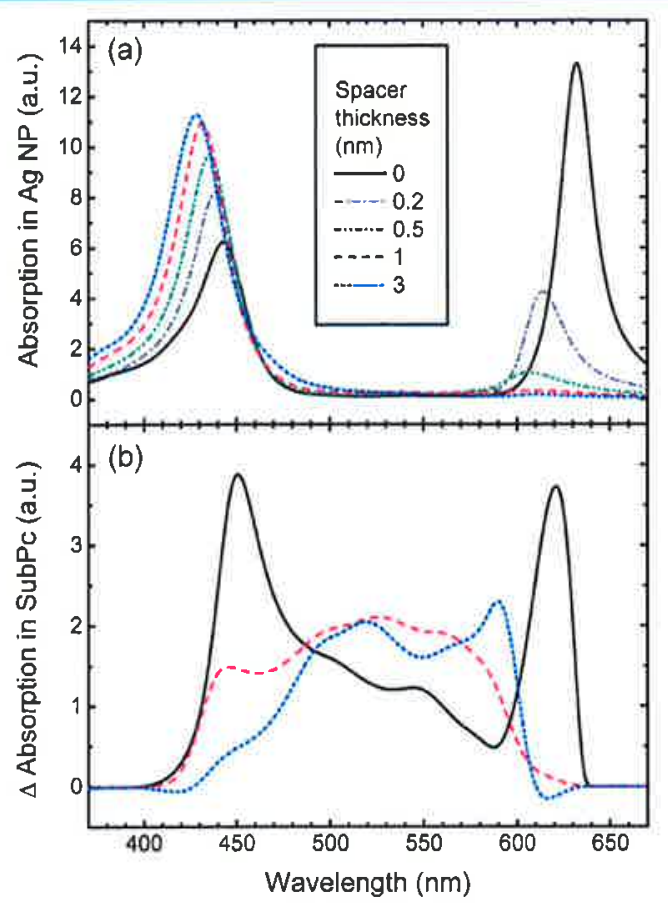

Figure 9. (a) Simulated absorption spectra in the Ag NPs when covered by a SubPc layer with $t$ equivalent to a $10 \mathrm{~nm}$ thick neat layer, with and without a conformal spacer layer of Hyflon AD60X separating the NPs from the SubPc layer. (b) Difference between the absorption in the SubPc layer covering the Ag NPs and a $10 \mathrm{~nm}$ thick neat SubPc layer, with and without Hyflon AD60X spacer layer.

at $\lambda=445$ and $630 \mathrm{~nm}$, respectively, due to the two dipole LSPR. These two bands lead to strong absorption enhancement in the SubPc layer at these wavelengths. The intensity of the $\mathrm{Ag}$ $\mathrm{NP}$ absorption band at $\lambda=630 \mathrm{~nm}$ rapidly decreases with increasing spacer layer thickness and completely vanishes at a spacer thickness of $1 \mathrm{~nm}$. The increase in absorption in the SubPc layer, on the other hand, remains for all simulated spacer layer thicknesses and features the shoulder-to-peak transition around $\lambda=600 \mathrm{~nm}$ observed experimentally (see Figure $8 \mathrm{~b}$ ).

For both CuPc and SubPc, the threshold spacer thickness for absorption enhancement is between 3 and $5 \mathrm{~nm}$, which is about the same as the Ag NP height. These results confirm that the absorption enhancement occurs within a few nanometers from the Ag NP surface independent of the embedding medium. In addition, the effect of $\varepsilon_{\mathrm{m}}$ on the absorption enhancement can indirectly be observed. By increasing the thickness of a spacer layer with a low $\varepsilon$, the effective $\varepsilon_{\mathrm{m}}$ gradually decreases from that of the initial organic medium to that of the spacer layer. For CuPc with $\varepsilon_{1}<4.5$, this transition is not too dramatic. For SubPc with $\varepsilon_{1}$ values of up to 10.5 , on the other hand, it leads to large changes in the spectral shape. Especially, the excitation of the LSPR at $\lambda=610 \mathrm{~nm}$ is extremely sensitive to the embedding medium and is suppressed by a spacer layer of only $1 \mathrm{~nm}$. The LSPR at $\lambda=445 \mathrm{~nm}$ on the other hand is much less effected by the spacer layer as at this wavelength the $\varepsilon$ of SubPc is similar to that of Hyflon AD60X. It is noteworthy that the simulated absorption in the $\mathrm{Ag} \mathrm{NPs}$ for a spacer layer thickness of $0.2 \mathrm{~nm}$ describes the experimentally obtained absorption 
difference for SubPc without a spacer layer much more accurately than the simulation without a spacer layer. As already discussed for the numerical simulation of the $\mathrm{Ag}$ NPs embedded in $\mathrm{CuPc}$, this distance roughly corresponds to the distance between the Ag surface and the first molecular adlayer (see Section 3.2). For SubPc, where the low energy LSPR strongly depends on $\varepsilon_{\mathrm{m}}$, the presence of this small gap makes a considerable difference.

\section{CONCLUSIONS}

We studied the absorption of thin-films comprising Ag NPs that show negligible far-field scattering embedded in molecular thin-films by means of experiment, numerical simulations, and analytical calculations. For $\mathrm{CuPc}$, we observed an increased intensity of the absorption $\mathrm{Q}$ band due to the $\mathrm{Ag}$ NP layer with absorption enhancement values of up to $1.44 \pm 0.08$ at $\lambda=690$ $\mathrm{nm}$ for an $8 \mathrm{~nm}$ thick CuPc layer. A strong dependence of this absorption enhancement on the CuPc layer thickness was found. We could quantitatively reproduce these experimental results by numerical simulations. From these simulations, the absorption enhancement was found to mainly occur in the $\mathrm{CuPc}$ medium, caused by an enhanced $\mathbf{E}$ field intensity in the interstices of the Ag NPs. The short range of this enhancement was confirmed experimentally by introducing a transparent spacer layer between the NPs and the CuPc layer, which led to a rapid decrease of the absorption enhancement with increasing spacer thickness. By employing an analytical model, the spectral shape of the $\mathrm{CuPc}$ absorption enhancement could be directly linked to the $\varepsilon$ values of the NP material and the embedding medium, while neglecting particle-particle and particlesurface interactions. We studied the effect of $\varepsilon_{\mathrm{m}}$ on the plasmon-exciton interactions by means of numerical simulations of materials with spectrally independent $\varepsilon$ and found absorption enhancement at $\lambda>\lambda_{\text {LSPR }}$ which extends to the near-infrared for $\varepsilon$ values typical of molecular media. When introducing a low-index spacer between the $\mathrm{Ag}$ NPs and a layer of SubPc, we observed a complex behavior of the spectral shape of the absorption enhancement due to the Ag NPs, which we attribute to the decrease of the effective $\varepsilon_{\mathrm{m}}$ with increasing spacer thickness.

Our results show that care has to be taken on both the geometrical features and the material choice when designing metal NP-organic molecule structures with the aim to enhance the absorption in the organic medium. For example, by coating metal NPs with a transparent layer of only a few nanometers, the absorption enhancement in an absorbing embedding molecular medium due to the NPs can be strongly affected and perhaps eliminated completely. In spite of the complex behavior of the plasmon-exciton interactions, we show that, at least in the case of $\mathrm{CuPc}$ and SubPc, the absorption enhancement in the embedding molecular medium due to metal NPs can be predicted accurately by numerical simulations that do not take the organic layer morphology into account. Furthermore, if the NPs are well separated, a simple analytical formula provides quantitative information about the spectral shape of the absorption enhancement. In part III, ${ }^{37}$ we will build upon the knowledge gained in this study and extend our work on plasmon-exciton interactions to the effect of the Ag NP layer on the emission of organic molecules.

\section{ASSOCIATED CONTENT}

\section{Supporting Information}

Comparison between the total and specular transmission spectra, absorption spectra of several organic layers with identical nominal thickness deposited during a single run, scattering and absorption cross section of a spherical Ag NP according to Mie theory, $\varepsilon$ values of all material used in this study, and absorption spectra of Hyflon AD60X thin-films deposited on glass and on the Ag NP layer. This material is available free of charge via the Internet at http://pubs.acs.org.

\section{AUTHOR INFORMATION}

\section{Corresponding Author}

*E-mail: Barry.Rand@imec.be.

\section{Notes}

The authors declare no competing financial interest.

\section{ACKNOWLEDGMENTS}

We gratefully acknowledge Josine Loo for obtaining scanning electron micrographs. We thank Robert Gehlhaar for helpful discussions, and Solvay-Solexis for providing Hyflon AD60X. This work was supported by the European Community's Seventh Framework Programme under grant no. FP7-ICT2009-4-248154 ("PRIMA"), the Institute for the Promotion of Innovation by Science and Technology in Flanders (IWT) via the SBO project no. 060843 ("PolySpec"), the Belgian Interuniversity Attraction Poles program under grant no. LAP P6-10 ("photonics@be"), and COST action MP 0702. P.V.D. thanks the Fonds Wetenschappelijk Onderzoek Vlaanderen (FWO) for financial support.

\section{REFERENCES}

(1) Kreibig, U.; Vollmer, M. Optical Properties of Metal Clusters; Springer: Berlin, Germany, 1995.

(2) Henry, A. I.; Bingham, J. M.j Ringe, E.; Marks, L. D.; Schatz, G. C,; Van Duyne, R. P. J. Phys. Chem. C 2011, 115, 9291-9305.

(3) Rycenga, M.; Cobley, C. M.; Zeng, J.; Li, W.; Moran, C. H.; Zhang, Q.; Qin, D.; Xia, Y. Chem. Rev. 2011, 111, 3669-3712.

(4) Tcherniak, A.; Ha, J. W.; Dominguez-Medina, S.; Slaughter, L. S.; Link, S. Nano Lett. 2010, 10, 1398-1404.

(5) Noguez, C. J. Phys. Chem. C 2007, 111, 3806-3819.

(6) Jain, P. K.; Lee, K. S.; El-Sayed, I. H.; El-Sayed, M. A. J. Phys. Chem. B 2006, 110, 7238-7248.

(7) Muskens, O.; Billaud, P.; Broyer, M.; Del Fatti, N.; Vallée, F. Phys. Rev. B 2008, 78, 205410.

(8) Miller, M. M.; Lazarides, A. A. J. Opt. A: Pure Appl. Opt. 2006, 8, S239-249.

(9) Maier, S. A. Plasmonics: Fundamentals and Applications; Springer: New York, 2007.

(10) Tanabe, K. J. Phys. Chem. C 2008, 112, 15721-15728.

(11) Fort, E.; Grésillon, S. J. Phys. D: Appl. Phys. 2008, 41, 013001

(12) Gersten, J.; Nitzan, A. J. Chem. Phys. 1981, 75, 1139-1152.

(13) Härtling, T.; Reichenbach, P.; Eng, L. M. Opt. Express 2007, 15, 12806-12817.

(14) Atwater, H. A.; Polman, A. Nat. Mater. 2010, 9, 205-213.

(15) Dunbar, R. B.; Pfadler, T.; Schmidt-Mende, L. Opt. Express 2012, 20, A177-A189.

(16) Niesen, B.; Rand, B. P.j Van Dorpe, P.; Cheyns, D.; Tong, L.; Dmitriev, A; Heremans, P. Adv. Energy Mater. 2012, DOI: 10.1002/ aenm.201200289.

(17) Diukman, I.; Tzabari, L.; Berkovitch, N.; Tessler, N.; Orenstein, M. Opt. Express 2011, 19, A64-71.

(18) Qi, J.; Dang, X.; Hammond, P. T.; Belcher, A. M. ACS Nano 2011, 5, 7108-7116. 
(19) Brown, M. D.; Suteewong, T.; Kumar, R S. S.; D'Innocenzo, V.; Petrozza, A.; Lee, M. M.; Wiesner, U.; Snaith, H. J. Nano Lett. 2011, $11,438-445$.

(20) Choulis, S. A.; Mathai, M. K.; Choong, V.-E. Appl. Phys, Lett. 2006, 88, 213503.

(21) Fujiki, A.; Uemura, T.; Zettsu, N.; Akai-Kasaya, M.j Saito, A. Kuwahara, Y. Appl. Phys. Lett. 2010, 96, 043307.

(22) Mayer, K. M.; Hafner, J. H. Chem. Rev. 2011, 111, 3828-3857.

(23) Haick, H. J. Phys. D: Appl. Phys. 2007, 40, 7173-7186.

(24) De, M.; Ghosh, P. S.; Rotello, V. M. Adv. Mater. 2008, 20, 4225-4241.

(25) Schlücker, S. ChemPhysChem 2009, 10, 1344-1354.

(26) Han, X. X; Zhao, B.j Ozaki, Y. Anal. Bioanal. Chem. 2009, 394, 1719-1727.

(27) Mori, K.; Kawashima, M.; Che, M.; Yamashita, H. Angew. Chem., Int. $E d .2010,49,8598-8601$.

(28) Noginov, M. A; Zhu, G.; Belgrave, A. M.; Bakker, R; Shalaev, V. M.; Narimanov, E. E.; Stout, S.; Herz, E.; Suteewong, T.; Wiesner, U. Nature 2009, 460, 1110-1112.

(29) Kéna-Cohen, S.; Wiener, A.j Sivan, Y.; Stavrinou, P. N.; Bradley, D. D. C.; Horsfield, A.; Maier, S. A. ACS Nano 2011, S, 9958-9965. (30) Rand, B. P.; Peumans, P.; Forrest, S. R. J. Appl. Phys. 2004, 96, 7519.

(3I) Kulkarni, A. P.; Noone, K. M.; Munechika, K; Guyer, S. R; Ginger, D. S. Nano Lett. 2010, 10, 1501-1505.

(32) Shen, H.; Bienstman, P.; Maes, B. J. Appl. Phys. 2009, 106, 073109.

(33) Khurgin, J. B.; Sun, G. J. Opt. Soc. Am. B 2009, 26, B83-95.

(34) Chen, Y.; Munechika, K; Ginger, D. S. Nano Lett. 2007, 7, $690-696$.

(35) Bharadwaj, P.; Novotny, L. Opt. Express 2007, 15, 1426614274.

(36) Cheng, Y.; Stakenborg, T.; Van Dorpe, P.; Lagae, L.; Wang, M.; Chen, H.j Borghs, G. Anal. Chem. 2011, 83, 1307-1314.

(37) Niesen, B.; Rand, B. P.; Van Dorpe, P.; Cheyns, D.; Fron, E.j Van der Auweraer, M.; Heremans, P. J. Phys. Chem. C 2012, DOI: $10.1021 /$ jp3058965.

(38) Nason, T. C.; Moore, J. A.; Lu, T.-M. Appl. Phys. Lett. 1992, 60, 1866-1868.

(39) Niesen, B.; Rand, B. P.; Van Dorpe, P.; Shen, H.; Maes, B.; Genoe, J.; Heremans, P. Opt. Express 2010, 18, 19032-19038.

(40) Kreibig, U.; Schmitz, B.; Breuer, H. D. Phys. Rev. B 1987, 36, 5027-5030.

(41) See, K C.; Spicer, J. B.; Brupbacher, J.; Zhang, D.; Vargo, T. G. J. Phys. Chem. B 2005, 109, 2693-2698.

(42) Bohren, C. F.; Huffman, D. R. Absorption and Scattering of Light by Small Particles; Wiley Interscience: New York, 1983.

(43) Lee, J.-Y.; Peumans, P. Opt. Express 2010, 18, 10078-10087.

(44) Malinsky, M. D.; Kelly, K. L.; Schatz, G. C.; Van Duyne, R. P. J. Am. Chem. Soc. 2001, 123, 1471-1482.

(45) Mock, J. J.; Smith, D. R; Schultz, S. Nano Lett. 2003, 3, 485491.

(46) Song, F.j Huang, H.j Dou, W.; Zhang, H.; Hu, Y.; Qian, H.; Li, H.; He, P.; Bao, S.; Chen, Q.; Zhou, W. J. Phys.: Condens. Matter 2007, 19, 136002.

(47) Rand, B. P.; Xue, J.j Uchida, S.; Forrest, S. R. J. Appl. Phys. 2005, 98,124902 .

(48) $\eta$ depends on the angle between the $\mathrm{E}$ and $\mathbf{r}$ vectors. It is maximized when these vectors are parallel (Hence, the reduction of $r$ to a scalar variable in eq 2 ).

(49) Lagos, N.; Sigalas, M. M.; Lidorikis, E. Appl. Phys. Lett. 2011, 99, 063304.

(50) Romero, I.; Aizpurua, J.; Bryant, G. W.; Garcia De Abajo, F. J. Opt. Express 2006, 14, 9988-9999.

(51) Khurgin, J. B.; Sun, G.; Soref, R. A. Appl. Phys. Lett. 2009, 94, 071103. 